\title{
Midbrain-Hindbrain Involvement in Septo-Optic Dysplasia
}

\author{
M. Severino, A.E.M. Allegri, A. Pistorio, B. Roviglione, N. Di lorgi, M. Maghnie, and A. Rossi
}

\begin{abstract}
BACKGROUND AND PURPOSE: Midbrain-hindbrain involvement in septo-optic dysplasia has not been well described, despite reported mutations of genes regulating brain stem patterning. We aimed to describe midbrain-hindbrain involvement in patients with septo-optic dysplasia and to identify possible clinical-neuroimaging correlations.
\end{abstract}

MATERIALS AND METHODS: Using MR imaging, we categorized 38 patients (21 males) based on the presence (group A, 21 patients) or absence (group B, 17 patients) of visible brain stem anomalies. We measured height and anteroposterior diameter of midbrain, pons, and medulla, anteroposterior midbrain/pons diameter (M/P ratio), vermian height, and tegmento-vermian angle, and compared the results with 114 healthy age-matched controls. Furthermore, patients were subdivided based on the type of midline anomalies. The associations between clinical and neuroradiological features were investigated. Post hoc tests were corrected according to Bonferroni adjustment ( $\mathrm{P}_{\mathrm{B}}$ ).

RESULTS: Patients with brain stem abnormalities had smaller anteroposterior pons diameter than controls $\left(\mathrm{p}_{\mathrm{B}}<.0001\right)$ and group $\mathrm{B}\left(\mathrm{p}_{\mathrm{B}}=\right.$ $.012)$, higher $M / P$ ratio than controls $\left(p_{B}<.0001\right)$ and group $B\left(p_{B}<.0001\right)$, and smaller anteroposterior medulla diameter $\left(p_{B}=.001\right)$, pontine height $\left(\mathrm{p}_{B}=.00072\right)$, and vermian height $\left(\mathrm{p}_{B}=.0009\right)$ than controls. Six of 21 patients in group $A$ had thickened quadrigeminal plate, aqueductal stenosis, and hydrocephalus; 3 also had agenesis of the epithalamus. One patient had a short midbrain with long pons and large superior vermis. There was a statistically significant association between brain stem abnormalities and callosal dysgenesis $(P=.011)$ and developmental delay $(P=.035)$, respectively.

CONCLUSION: Midbrain-hindbrain abnormalities are a significant, albeit underrecognized, component of the septo-optic dysplasia spectrum, and are significantly associated with developmental delay in affected patients.

ABBREVIATIONS: $\mathrm{AP}=$ anteroposterior; $\mathrm{MH}=$ midbrain-hindbrain; $\mathrm{SOD}=$ septo-optic dysplasia

$\mathbf{S}_{\mathrm{sp}}^{\mathrm{pp}}$ epto-optic dysplasia (SOD) is a highly heterogeneous, usually sporadic condition with a multifactorial etiology. Currently, genetic abnormalities are identified only in $1 \%$ of patients, whereas the etiology remains unclear in most. ${ }^{1}$ However, an increasing number of early developmental transcription factors and associated pathway genes have been implicated in the etiology of SOD, ie, HESX1, SOX2, SOX3, OTX2, PROKR2, FGF1, and

Received November 11, 2013; accepted after review January 21, 2014.

From the Neuroradiology Unit (M.S., A.R.), Pediatric Department (A.E.M.A., N.D.I., M.M.), and Epidemiology and Biostatistics Unit (A.P.), Istituto Giannina Gaslini, Università di Genova, Genoa, Italy; and Università di Genova (B.R.), Genoa, Italy.

Paper previously presented at: Annual Meeting of the American Society of Neuroradiology, May 18-23, 2013; San Diego, California.

Please address correspondence to Mariasavina Severino, Department of Neuroradiology, Istituto Giannina Gaslini, via Gaslini 5, 16147 Genova, Italy, e-mail: mariasavinaseverino@ospedale-gaslini.ge.it

三 Indicates article with supplemental on-line tables.

http://dx.doi.org/10.3174/ajnr.A3959
FGF8. ${ }^{2-4}$ These genes are expressed in regions that determine the formation of forebrain and related midline structures, such as the hypothalamus and pituitary gland, and mutations in these genes are therefore associated with marked phenotypic heterogeneity. Classically, the diagnosis of SOD is made when 2 or more features of the classic triad of optic nerve hypoplasia, pituitary hormone abnormalities, and midline brain defects are present. ${ }^{1}$ However, only one-third of patients present with all cardinal features of SOD. ${ }^{3}$ Visual deficits due to optic nerve hypoplasia and ocular malformations are usually the first presenting sign, whereas endocrine dysfunction may become apparent later on. ${ }^{1}$ The severity of pituitary-hypothalamic dysfunction is highly variable, ranging from isolated deficit of pituitary hormones to panhypopituitarism. ${ }^{3}$ Neurologic deficits are common, and range from global retardation to focal deficits such as epilepsy or hemiparesis. ${ }^{5}$

Interestingly, OTX2 and FGF8 are also pivotal genes regulating brain stem patterning during early embryologic development. ${ }^{6-9}$ In recent years, there has been a growing interest in the descrip- 

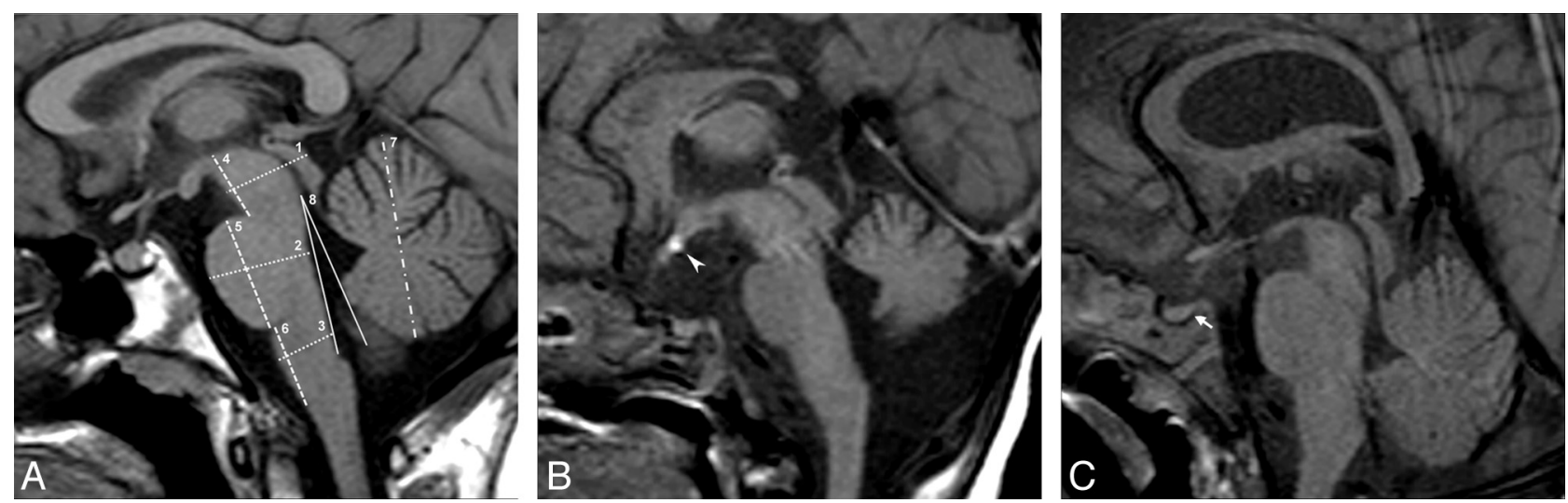

FIG 1. A, Midsagittal Th-weighted image in a healthy child demonstrates how the measurements were performed: dotted lines indicate the AP diameter of the midbrain (1), pons (2), and medulla (3), dashed lines indicate the height of the midbrain (4), pons (5), and medulla (6), dash-dot line indicates the height of the vermis (7), and solid lines indicates the tegmento-vermian angle (8). B, Midsagittal TT-weighted image in a patient with SOD with visible brain stem abnormalities (group A) reveals hypoplasia of the pons and cerebellar vermis. Note the hypoplasia of the pituitary gland with ectopic posterior lobe (arrowhead) and associated small corpus callosum. C, Midsagittal TT-weighted image in a patient with SOD without visible brain stem abnormalities (group B) reveals grossly normal brain stem and vermis. Note the absence of the normal posterior pituitary bright spot (arrow).

tion of congenital brain stem abnormalities. However, midbrainhindbrain $(\mathrm{MH})$ involvement in SOD has not been described so far. Here, we aimed to describe midbrain abnormalities and to identify possible correlations with clinical features in a relatively large group of patients with SOD.

\section{METHODS}

\section{Subjects}

This was a single-center retrospective case-control study. We reviewed the MR studies of 38 children who were diagnosed with SOD between 2006 and 2012 (21 males, mean age $61.8 \pm 60.7$ months, and age range from 2 days to 18.3 years at the time of scan). The study received institutional review board approval. Exclusion criteria were uncertain SOD diagnosis and poor-quality or incomplete images. Complete neurologic evaluation was available in 32 of 38 patients. Griffiths Mental Development and Binet-Simon scales were used to define the developmental delay. Karyotype was performed in all patients. One patient harbored a mosaicism of trisomy chromosome 8 in skin fibroblast karyotypes, whereas the remainder presented normal karyotype. Comparative genomic hybridization by microarray was performed in 12 out of 38 patients, revealing de novo chromosomal aberrations in 2 cases: 1 duplication at 16p13.3p13.13 and 1 deletion at 14q22.1q23.1.

We selected 114 age-and sex-matched healthy children as a control group, ie, 3 controls for every patient with SOD. Controls underwent brain MR imaging for transient febrile convulsion or headaches. All were diagnosed as neurologically and developmentally normal by pediatric neurologists and had normal MR imaging findings.

\section{Imaging Analysis}

All MR imaging examinations were performed on a $1.5 \mathrm{~T}$ magnet (Intera Achieva 2.6; Philips, Best, the Netherlands). Children under 6 years of age or uncooperative patients were sedated during examinations. All MR imaging studies included 3-mm-thick sagittal spin-echo T1-weighted images, axial and coronal FSE T2weighted images, axial FLAIR images, and axial DWI. Additional sequences performed in patients with SOD included axial and sagittal 0.6-mm-thick 3D driven equilibrium sequences, sagittal FSE T2-weighted images, and 3D turbo-field-echo T1-weighted images.

Patients with SOD were subjectively categorized based on the presence (group A, 21 patients) or absence (group B, 17 patients) of visible brain stem anomalies. Systematic visual analysis included midbrain dorsoventral and rostrocaudal size, tectal thickness, and collicular morphology, as well as dorsoventral and rostrocaudal size of the pons and medulla oblongata, and vermian size. We then measured the height and anteroposterior (AP) diameter of midbrain, pons, and medulla, the vermian height, and the tegmento-vermian angle (Fig 1A). All measurements were performed by one pediatric neuroradiologist (M.S.) on midline sagittal T1-weighted images. We calculated the ratio of AP midbrain diameter to AP pons diameter ( $\mathrm{M} / \mathrm{P}$ ratio). We then compared the measurements and the $\mathrm{M} / \mathrm{P}$ ratios of patients with SOD versus the controls.

Patients with SOD were also subdivided based on the presence and type of midline anomalies into 3 subtypes: group I (septal agenesis, 23 patients; complete in 13 cases and partial in 10 cases); group II (abnormal corpus callosum, 7 patients); and group III (no midline brain defects, 8 patients). Then, we investigated the association between brain stem and midline abnormalities.

Images were also analyzed for abnormalities involving the eyes, optic nerves, pituitary gland, olfactory bulbs, hypothalamus and hippocampi, as well as for cortical malformations including polymicrogyria, periventricular nodular heterotopia, and schizencephaly. Optic nerve hypoplasia was evaluated on coronal T2weighted images at the level of the intraorbital segment, and was defined as an optic nerve caliber below $2 \mathrm{~mm}$. Finally, we studied the association between brain stem abnormalities and the presence or absence of pituitary gland abnormalities, hippocampal malrotation, cortical malformations, developmental delay (irrespective of the degree of cognitive impairment), and epilepsy.

\section{Statistics}

Descriptive statistics were firstly performed: categoric variables were reported in terms of absolute frequencies and percentages; 

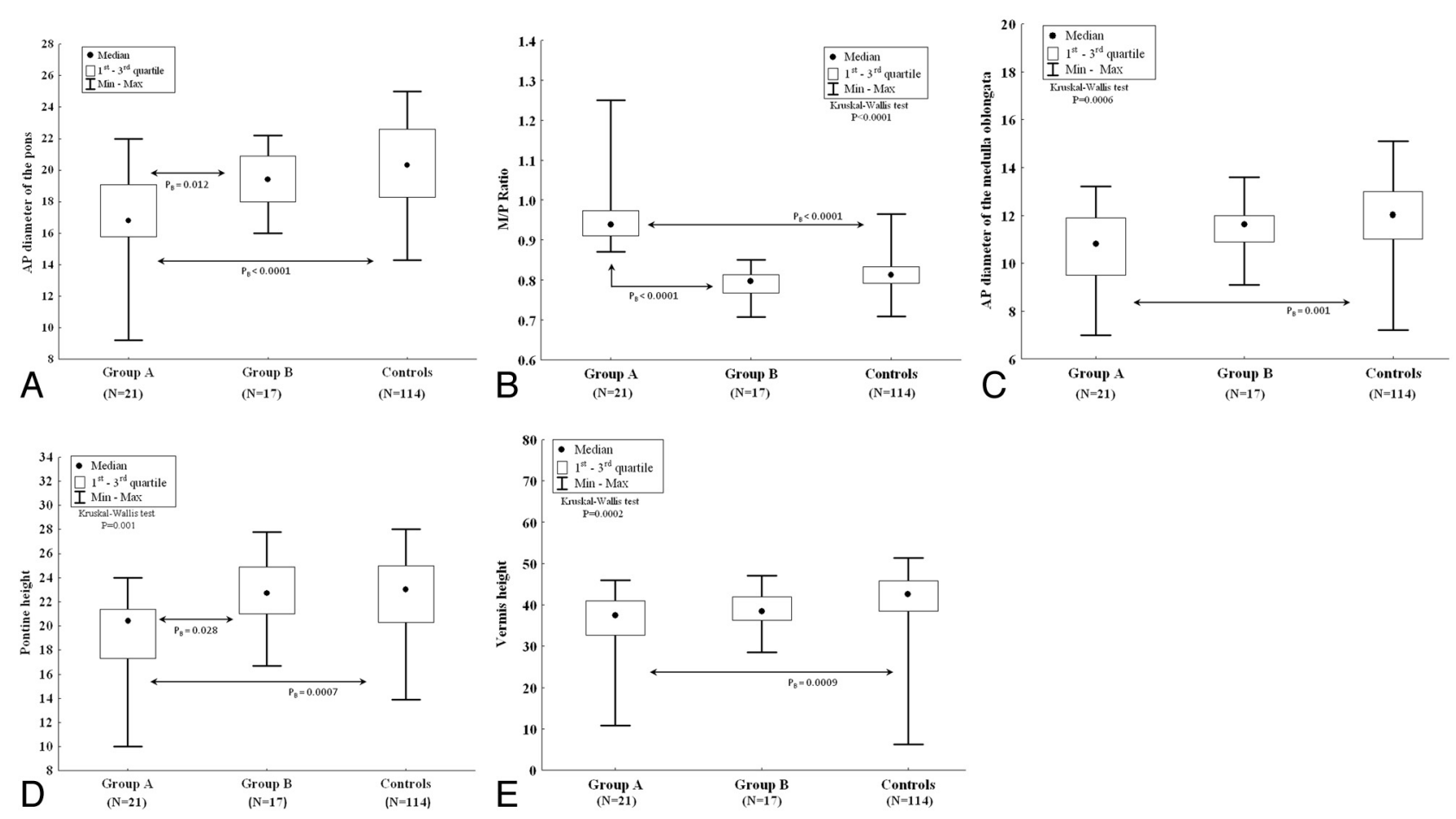

FIG 2. Boxplots of MH measurements in patients with SOD (group $A$ and $B$ ) and healthy controls. AP diameter of the pons $(A)$ and medulla $(B)$, midbrain to pons ratio $(C)$, height of the pons $(D)$, and vermis $(E)$ are shown.

quantitative variables were reported in terms of median values with 1st-3rd quartiles. Differences between groups were evaluated by means of the nonparametric ANOVA (Kruskal-Wallis test) and post hoc tests were corrected according to Bonferroni adjustment $\left(\mathrm{p}_{\mathrm{B}}\right)$. The association between categoric variables was evaluated by $\chi^{2}$ test, or by the Fisher exact test in case of expected frequencies $<5$. All tests were 2 -sided and a $P$ value less than .05 was considered as statistically significant. Data were analyzed by means of the statistical software Statistica 9 (StatSoft Corporation, Tulsa, Oklahoma) and STATA 7 (StataCorp, College Station, Texas).

\section{RESULTS}

Clinical and neuroradiologic characteristics of patients with SOD are described in On-line Table 1. The AP pontine diameter in patients with SOD with brain stem abnormalities (group A, Fig. $1 B$ ) was significantly smaller (median: $16.8 \mathrm{~mm}$; 1st-3rd quartiles: $15.8-19.1$ ) than in the control group (median: $19.4 \mathrm{~mm}$; 1st-3rd quartiles: $17.8-22.2, \mathrm{p}_{\mathrm{B}}<.0001$ ) and in patients with SOD with normal-appearing brain stem (group B, Fig 1C) (median: $19.4 \mathrm{~mm}$; 1st-3rd quartiles: $\left.18.0-20.9, \mathrm{p}_{\mathrm{B}}=.012\right)($ Fig $2 \mathrm{~A})$. No significant statistical difference was found in the AP diameter of midbrain between patients with SOD (both group A and B) and healthy controls. As a consequence, the M/P ratio was higher in patients with SOD with visible brain stem abnormalities (group A, median: 0.94; 1st-3rd quartiles: 0.91-0.97) compared with controls (median: 0.81; 1st-3rd quartiles: 0.79-0.84, $\mathrm{p}_{\mathrm{B}}<.0001$ ) and to group B (median: $0.80 ; 1$ st-3rd quartiles: $0.77-0.81, \mathrm{p}_{\mathrm{B}}<$ .0001) (Fig 2B).

The AP diameter of the medulla in patients with SOD with brain stem abnormalities (group A) was significantly smaller (median: $10.8 \mathrm{~mm}$; 1st-3rd quartiles: 9.5-11.9) (Fig. 1B) than in the control group (median $11.8 \mathrm{~mm}$; 1st-3rd quartiles: 10.6-13, $\mathrm{p}_{\mathrm{B}}=$ .001) (Fig 2C).

The height of the pons (median: $20.4 \mathrm{~mm}$; 1st-3rd quartiles: 17.3-21.4) and of the vermis (median: $37.4 \mathrm{~mm}$; 1 st-3rd quartiles: 32.7-41.0) was significantly shorter in patients with SOD with visible brain stem abnormalities (group A) than in controls (median: $22.0 \mathrm{~mm}$; 1st-3rd quartiles: 19.3-25.0, $\mathrm{p}_{\mathrm{B}}=.0007$ and median: $41.4 \mathrm{~mm}$; 1st-3rd quartiles: $36.3-45.0, \mathrm{p}_{\mathrm{B}}=.0009$, respectively) (Fig 2D, -E).

In patients of group A, the AP diameter of the pons and medulla and the pontine and vermian height were lower compared with the values observed in control subjects of the same age category (Fig 3); in patients of group B, pontine and vermis height values tended to be lower compared with the values of control subjects of the same age category but these differences did not reach statistical significance.

No significant statistical difference was found regarding the tegmento-vermian angle and height of midbrain and medulla between patients with SOD (both group A and B) and controls. There were no patients with isolated vermis anomalies.

Six of 21 patients $(28.6 \%)$ with brain stem abnormalities (group A) showed thickening of the quadrigeminal plate with aqueductal stenosis and triventricular hydrocephalus (Fig 4A); 3 also had agenesis of the epithalamus (Fig $4 B,-C$ ).

Callosal dysgenesis was present in 7 patients with SOD (group II) with complete agenesis in 3 cases and partial agenesis in 4 cases. Brain stem abnormalities were more frequent in patients with callosal dysgenesis $(7 / 21,33.3 \%)$ compared with those without visible brain stem abnormalities $(0 / 17,0 \%)(P=.011$, On-line Table 2).

Finally, in 1 patient harboring 14q22.1-q23.1 deletion the 

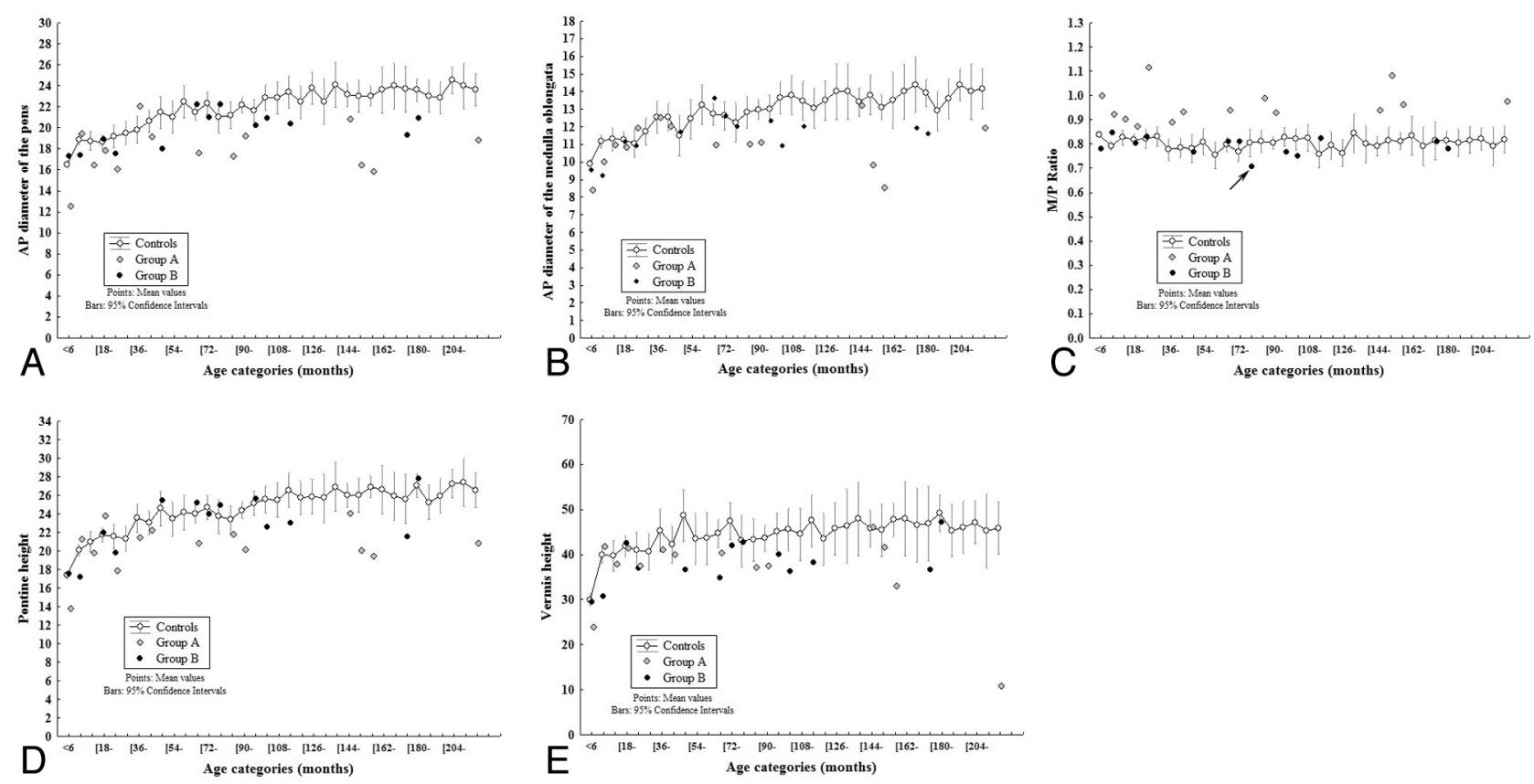

FIG 3. Scatterplots of MH measurements for age categories in patients with SOD and healthy controls. AP diameter of the pons (A) and medulla $(B)$, midbrain to pons ratio $(C)$, height of the pons $(D)$, and vermis $(E)$ are shown. In healthy controls, the AP diameters and height of brain stem structures and vermis showed an exponential growth curve. The growth spurt was steep until 16 months of age. Thereafter, it became less steep, reaching the adult level at about 8 years of age. On the other hand, the M/P ratios remained stable in controls over time, with a mean value of 0.82. In patients of group A, the AP diameter of the pons and medulla and the pontine and vermian height were lower compared with the values observed in control subjects of the same age category; in patients of group B, pontine and vermis height values tended to be lower compared with the values of control subjects of the same age category but these differences did not reach statistical significance. C, Note the lower M/P ratio in the patient harboring the 14q22.1-q23.1 deletion (arrow) compared with the corresponding values in age-matched controls.
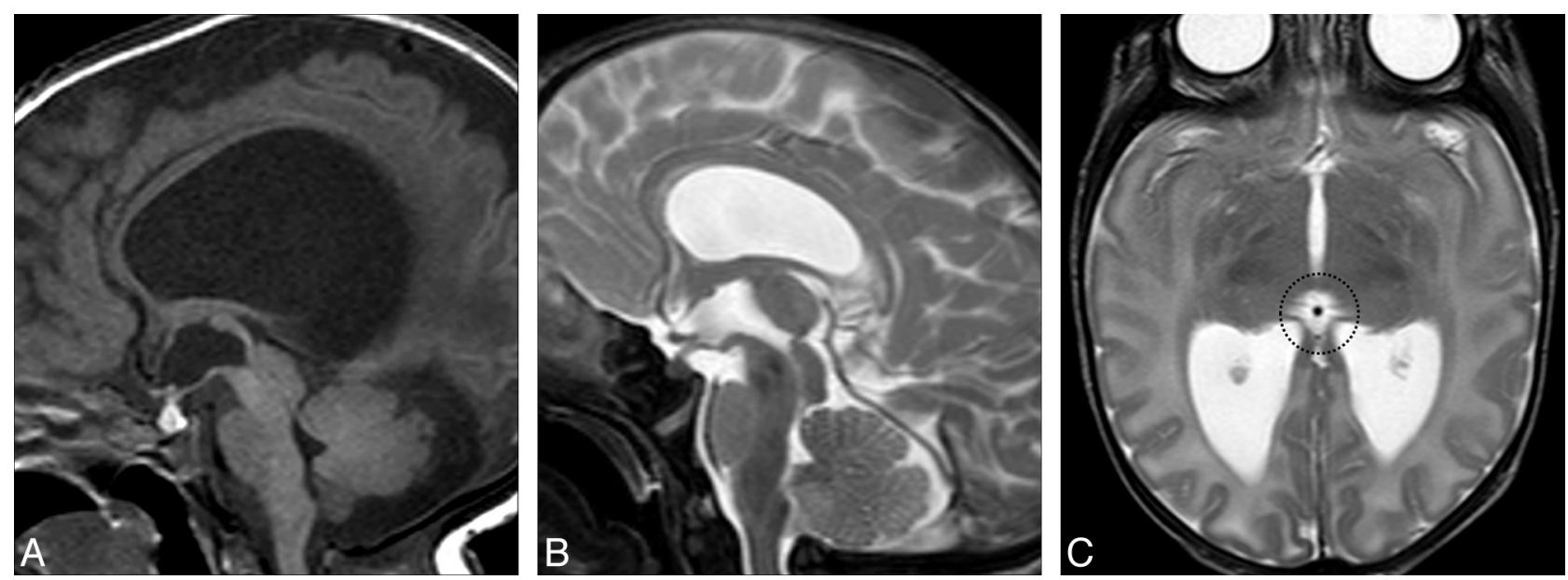

FIG 4. A, Midline sagittal T1-weighted image demonstrates hypoplasia of the pons and small vermis, and enlarged quadrigeminal plate with secondary aqueductal stenosis and triventricular hydrocephalus. Midline sagittal (B) and axial (C) T2-weighted images in another patient reveal agenesis of the epithalamus including agenesis of the pineal gland (dotted circle), stria medullaris, and posterior commissure.

midbrain was smaller (height $11 \mathrm{~mm}$ and AP diameter $13.8 \mathrm{~mm}$ versus $13.5-16.2 \mathrm{~mm}$ and $16.6-17.2 \mathrm{~mm}$ minimal-maximal values of age-matched controls, respectively) compared with the pons, which appeared slightly elongated (pons height $25.4 \mathrm{~mm}$ versus 23.2-25.3 $\mathrm{mm}$ minimal-maximal values of age-matched controls) (Fig 5). The M/P ratio in this patient was lower (0.76) compared with the corresponding minimal-maximal values in age-matched controls $(0.78-0.81)$ (Fig 3C).

Twenty-six of 38 patients presented with pituitary hypoplasia (68.4\%) with additional ectopic posterior pituitary lobe in 7 cases and absent posterior bright spot in 2 cases. Hypothalamic hamar- tomas were additionally present in 2 cases, whereas hypothalamic dysgenesis was evident in 3 other patients. Twenty-seven of 38 patients presented with optic nerve hypoplasia (71\%) with microphthalmia in 3 cases and colobomas in 4 cases. Septal agenesis and optic nerve hypoplasia without any pituitary abnormality were present in 10 patients. Seventeen patients presented with hippocampal malrotation $(44.7 \%)$ whereas 14 patients presented with a cortical malformation $(36.8 \%)$. In particular, unilateral or bilateral polymicrogyria was present in 8 patients whereas 2 patients presented with an abnormal cortical gyration pattern. Periventricular nodular heterotopias were evident in 6 patients. Only 1 patient 


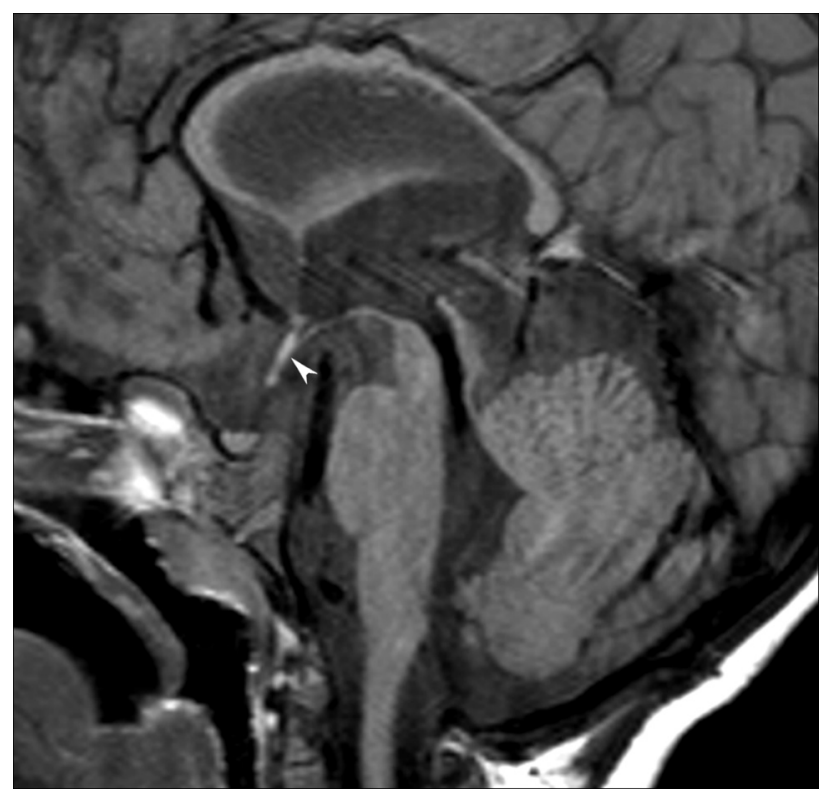

FIG 5. Midsagittal T1-weighted image of the patient harboring the 14q22.1-q23.1 deletion shows a small and short midbrain with elongated pons and relatively larger superior portion of the cerebellar vermis. Note the small anterior pituitary gland with ectopic posterior lobe along the pituitary stalk (arrowhead).

had a unilateral schizencephaly. The association between these neuroradiologic abnormalities and brain stem malformation are summarized in On-line Table 3. We found a statistically significant association between pituitary and brain stem anomalies $\left(P=.002, \chi^{2}\right)$. Seventeen of 32 patients (53.1\%) were developmentally delayed; 5 of 32 patients (15.6\%) had epilepsy. We found a statistically significant association between brain stem abnormalities and developmental delay $\left(P=.035 ; \chi^{2}\right)$.

\section{DISCUSSION}

The wide spectrum of imaging findings in SOD includes variable combinations of abnormalities of midline brain structures, the pituitary gland, optic nerves and eyes, olfactory bulbs, and other brain structures. ${ }^{10}$ Midline brain defects classically consist of complete or partial absence of the septum pellucidum with fused midline fornices ( $60 \%$ of cases) and/or corpus callosum abnormalities, such as agenesis, dysplasia, or hypoplasia. ${ }^{1,10,11}$ Pituitary gland malformations include anterior pituitary hypoplasia, ectopic posterior lobe, and/or thin or interrupted pituitary stalk. ${ }^{3} \mathrm{Op}-$ tic nerve hypoplasia is frequently associated with ocular anomalies such as coloboma, anophthalmia, and microphthalmia. ${ }^{12}$ The olfactory bulbs may be absent or hypoplastic. ${ }^{13}$ Other common associations include schizencephaly (so-called SODplus), gray matter heterotopia, polymicrogyria, and hippocampal malformations. ${ }^{14-16}$

$\mathrm{MH}$ abnormalities have not been described in patients with SOD to the best of our knowledge. Qualitative assessment of $\mathrm{MH}$ structures in the present study revealed abnormalities in $55 \%$ of patients, especially in the corpus callosum agenesis subgroup and in patients with pituitary gland abnormalities. Careful measurement and comparison of the height and AP diameter of midbrain, pons, medulla, and vermian height in patients with SOD versus healthy controls confirmed the visual analysis. Hypoplasia of the pons was the major finding, leading to a significantly higher midbrain to pons ratio compared with controls. Moreover, hypoplasia of the vermis and medulla was also associated, resulting in a globally small hindbrain. The term "hypoplasia" refers to a condition of incomplete or arrested development in which an organ or part of it remains below the normal size. Generalized or segmental brain stem hypoplasia may be an isolated malformative feature or part of a more complex $\mathrm{MH}$ malformation, such as pontocerebellar hypoplasia, ${ }^{17}$ horizontal gaze palsy with progressive scoliosis, ${ }^{18}$ pontine tegmental cap dysplasia, ${ }^{19}$ and anteroposterior or dorsoventral $\mathrm{MH}$ patterning defects. ${ }^{20,21}$ More frequently, brain stem hypoplasia is identified in patients with cerebral malformations such as congenital muscular dystrophies $^{22}$ lissencephalies, ${ }^{23}$ or cerebral commissural anomalies. ${ }^{20}$ As previously described in other $\mathrm{MH}$ malformations, ${ }^{24}$ we found that cognitive impairment or developmental delay was more frequent in patients with SOD with small hindbrain, worsening the prognosis and impairing rehabilitative strategies.

Recently, mutations in OTX2 and FGF8 genes have been described in patients with SOD. ${ }^{2-4}$ Interestingly, OTX2 and FGF8 play an important role both in the development of the anterior forebrain $^{25}$ and in the differentiation along the anteroposterior axis of the neural tube, with formation of the primary and secondary brain vesicles. ${ }^{26}$ The latter process determines the correct regionalization of the forebrain (which rapidly divides into telencephalon and diencephalon), mesencephalon (midbrain), and hindbrain (which further divides into the rostral metencephalon, ie, pons and cerebellum, and caudal myelencephalon, ie, medulla oblongata). This delicate embryologic process requires the orderly expression and interaction of several signaling molecules and the formation of patterning centers such as the isthmus organizer at the $\mathrm{MH}$ junction. ${ }^{27}$ In particular, Otx2 is expressed in the midbrain both in mice ${ }^{28}$ and humans, ${ }^{29}$ whereas Gbx2 is expressed in the anterior hindbrain, with a shared border at the level of IsO. ${ }^{30}$ In rodent fetuses, the formation and normal location of $\mathrm{IsO}$ depends on a delicate interplay between the expression of Otx2, Gbx2, ${ }^{6,7}$ and Fgf8. ${ }^{30}$ Animal models have shown that repression of Otx2 expression induces Gbx2 formation to establish the location of the $\mathrm{MH}$ junction rostrally, resulting in a short midbrain and long pons. On the other hand, overexpression of Otx2 shifts the MH junction caudally, resulting in an elongated midbrain with a small pons and vermis. ${ }^{27}$ Similarly, alterations of Pax6 or En1/Pax2 will change the location of the diencephalicmesencephalic junction. ${ }^{27}$

Remarkably, 6 patients with SOD spectrum and hypoplasia of hindbrain structures also showed hypertrophy of the mesencephalic tectum with consequent aqueductal stenosis and triventricular hydrocephalus. Broccoli et $\mathrm{al}^{6}$ assessed the effect of ectopic expression of Otx 2 on $\mathrm{MH}$ development in mutant mice, and noticed that the superior and inferior colliculi appeared enlarged and were shifted posteriorly in all analyzed mutants. Furthermore, 3 patients also showed evidence of agenesis of the epithalamus (ie, stria medullaris, habenular trigone, pineal gland, and posterior commissure). Larsen et $\mathrm{al}^{29}$ recently demonstrated that Otx2 is expressed in the pineal gland at later stages in human fetuses. Gene ablation experiments in mice revealed that Otx 2 is a key regulatory gene for the development of the pineal gland. ${ }^{31}$ 
Furthermore, agenesis of the pineal gland has been described in patients with mutations of PAX6 gene and congenital aniridia. ${ }^{32,33}$ Intriguingly, PAX6 is another important gene involved in the AP patterning of the brain. In particular, in murine and chick models, the correct formation of the diencephalic-mesencephalic junction depends on the interaction of Pax6 from the diencephalon and En1/Pax2 from the rostral mesencephalon, with the contributing role of FGf8 that induces forebrain cells to secrete Pax6. ${ }^{27}$ Webb et al ${ }^{34}$ recently studied the 24-hour melatonin profiles in 6 patients with SOD and sleep disorders. Interestingly, 2 children appeared to produce virtually no melatonin throughout the 24-hour period of measurement. These findings raise the suspicion of a possible correlation between pineal gland agenesis and abnormally reduced melanin production in patients with SOD, which awaits confirmation in prospective studies.

Finally, 1 patient in this study harboring a de novo deletion at 14q22.1-23.1 presented with a short, small midbrain with elongated pons and enlarged superior cerebellar vermis, suggesting an abnormality of $\mathrm{MH}$ anteroposterior patterning due to rostral misplacement of the IsO. ${ }^{20-21}$ This patient also had right anophthalmia and left colobomatous microphthalmia, small corpus callosum, bilateral hippocampal malformation, and hypoplasia of the anterior pituitary lobe with an ectopic posterior lobe. Interestingly, the 14q22.1-23.1 deletion includes the Otx2 gene, supporting the hypothesis that loss of Otx2 expression may determine rostral misplacement of the IsO also in humans. OTX2 heterozygous mutations or deletions in humans have been linked to severe ocular malformations associated with forebrain abnormalities such as hippocampal and callosal malformations, and combined or multiple pituitary hormone deficiencies. ${ }^{12}$ However, no careful description of $\mathrm{MH}$ structures has been provided so far in these patients. We reviewed the available brain MR midline sagittal images including the posterior cranial fossa of previously reported patients with Otx2 mutations ${ }^{35-38}$ or chromosome 14 deletions including Otx $2,{ }^{39-43}$ and we recognized a similar midbrain hypoplasia with a long pons and large superior vermis in 5 patients. $^{35,36,40,41,43}$ We therefore suggest that $\mathrm{MH}$ abnormalities may have been underestimated in patients with Otx 2 mutations or deletion; further studies on larger series are awaited to address this hypothesis.

This study has several limitations, mainly including its retrospective design, the relatively small sample size, and the incomplete genetic assessment. Furthermore, the functional role and clinical implications of $\mathrm{MH}$ abnormalities in patients with SOD remain uncertain, and require further prospective studies.

\section{CONCLUSION}

$\mathrm{MH}$ abnormalities are a significant, albeit under-recognized, component of the SOD spectrum. We demonstrated that the pons, medulla, and vermis were significantly hypoplastic in more than $50 \%$ of patients with SOD. Additional features included midbrain tectum enlargement and agenesis of the epithalamus. Patients with these abnormalities were more likely to present with developmental delay. Interestingly, these anomalies are similar to those reported in mutant mice with caudal shift of the MH junction due to ectopic Otx2 expression or Fgf8 mutations. Conversely, a short midbrain with an elongated pons and large superior vermis was observed in one SOD patient with 14q22.1-23.1 deletion, supporting the hypothesis that deletion of the Otx2 gene may determine a rostral shift of the $\mathrm{MH}$ also in humans.

\section{ACKNOWLEDGMENTS}

We are grateful to the patients and families for participating in the study.

Disclosures: Mariasavina Severino-RELATED: Support for Travel to Meetings for the Study or Other Purposes: Ricerca corrente-Ministero della Salute, Comments: Support for travel to the ASNR 51st Annual Meeting and The Foundation of the ASNR Symposium 2013, San Diego, CA; Travel/Accommodations/Meeting Expenses Unrelated to Activities Listed: Ricerca corrente-Ministero della Salute.

\section{REFERENCES}

1. Webb EA, Dattani MT. Septo-optic dysplasia. Eur J Hum Genet 2010;18:393-97

2. McCabe MJ, Gaston-Massuet C, Tziaferi V, et al. Novel FGF8 mutations associated with recessive holoprosencephaly, craniofacial defects, and hypothalamo-pituitary dysfunction. J Clin Endocrinol Metab 2011;96:1709-18

3. McCabe MJ, Alatzoglou KS, Dattani MT. Septo-optic dysplasia and other midline defects: the role of transcription factors: HESX1 and beyond. Best Pract Res Clin Endocrinol Metab 2011;25:115-24

4. Raivio T, Avbelj M, McCabe MJ, et al. Genetic overlap in Kallmann syndrome, combined pituitary hormone deficiency, and septo-optic dysplasia. J Clin Endocrinol Metab 2012;97:694-99

5. Signorini SG, Decio A, Fedeli C, et al. Septo-optic dysplasia in childhood: the neurological, cognitive and neuro-ophthalmological perspective. Dev Med Child Neurol 2012;54:1018-24

6. Broccoli V, Boncinelli E, Wurst W. The caudal limit of Otx2 expression positions the isthmic organizer. Nature 1999;401:164-68

7. Millet S, Campbell K, Epstein DJ, et al. A role for $\mathrm{Gbx} 2$ in repression of Otx2 and positioning the mid/hindbrain organizer. Nature 1999;401:161-64

8. Sato T, Joyner AL. The duration of Fgf8 isthmic organizer expression is key to patterning different tectal-isthmo-cerebellum structures. Development 2009;136:3617-26

9. Beby F, Lamonerie T. The homeobox gene Otx2 in development and disease. Exp Eye Res 2013;111:9-16

10. Severino M. Septo-optic dysplasia. In: Rumboldt Z, Castillo M, Huang B, Rossi A, eds. Brain Imaging With MRI and CT: An Image Pattern Approach. New York: Cambridge University Press; 2012:146-47

11. Belhocine O, André C, Kalifa G, et al. Does asymptomatic septal agenesis exist? A review of 34 cases. Pediatr Radiol 2005;35:410-18

12. Ragge NK, Brown AG, Poloschek CM, et al. Heterozygous mutations of OTX2 cause severe ocular malformations. Am J Hum Genet 2005;76:1008-22

13. Levine LM, Bhatti MT, Mancuso AA. Septo-optic dysplasia with olfactory tract and bulb hypoplasia. Journal of AAPOS 2001;5:398-99

14. Barkovich AJ, Fram EK, Norman D. Septo-optic dysplasia: MR imaging. Radiology 1989;171:189-92

15. Miller SP, Shevell MI, Patenaude Y, et al. Septo-optic dysplasia plus: a spectrum of malformations of cortical development. Neurology 2000;54:1701-03

16. Riedl S, Vosahlo J, Battelino T, et al. Refining clinical phenotypes in septo-optic dysplasia based on MRI findings. Eur J Pediatr 2008;167:1269-76

17. Namavar Y, Barth PG, Poll-The BT, et al. Classification, diagnosis and potential mechanisms in pontocerebellar hypoplasia. Orphanet J Rare Dis 2011;12:6:50

18. Rossi A, Catala M, Biancheri R, et al. MR imaging of brain-stem 
hypoplasia in horizontal gaze palsy with progressive scoliosis. AJNR Am J Neuroradiol 2004;25:1046-48

19. Briguglio M, Pinelli L, Giordano L, et al. Pontine tegmental cap dysplasia: developmental and cognitive outcome in three adolescent patients. Orphanet J Rare Dis 2011;6:36

20. Barkovich AJ, Millen KJ, Dobyns WB. A developmental classification of malformations of the brainstem. Ann Neurol 2007;62:625-39

21. Barkovich AJ, Millen KJ, Dobyns WB. A developmental and genetic classification for midbrain-hindbrain malformations. Brain 2009; 132:3199-230

22. Clement E, Mercuri E, Godfrey C, et al. Brain involvement in muscular dystrophies with defective dystroglycan glycosylation. Ann Neurol 2008;64:573-82

23. Jissendi-Tchofo P, Kara S, Barkovich AJ. Midbrain-hindbrain involvement in lissencephalies. Neurology 2009;72:410-18

24. Soto-Ares G, Joyes B, Lemaitre MP, et al. MRI in children with mental retardation. Pediatr Radiol 2003;33:334-45

25. Matsuo I, Kuratani S, Kimura C, et al. Mouse Otx2 functions in the formation and patterning of rostral head. Genes Dev 1995;9: $2646-58$

26. Acampora D, Gulisano M, Broccoli V, et al. Otx genes in brain morphogenesis. Prog Neurobiol 2001;64:69-95

27. Nakamura $H$, Watanabe $Y$. Isthmus organizer and regionalization of the mesencephalon and metencephalon. Int J Dev Biol 2005;49: 231-35

28. Simeone A, Acampora D, Gulisano M, et al. Nested expression domains of four homeobox genes in developing rostral brain. Nature 1992;358:687-90

29. Larsen KB, Lutterodt MC, Møllgård K, et al. Expression of the homeobox genes OTX2 and OTX1 in the early developing human brain. J Histochem Cytochem 2010;58:669-78

30 Liu A, Joyner AL. EN and GBX2 play essential roles downstream of FGF8 in patterning the mouse mid/hindbrain region. Development 2001;128:181-91

31. Nishida A, Furukawa A, Koike C, et al. Otx2 homeobox gene controls retinal photoreceptor cell fate and pineal gland development. Nat Neurosci 2003;6:1255-63

32. Abouzeid H, Youssef MA, ElShakankiri N, et al. PAX6 aniridia and interhemispheric brain anomalies. Mol Vis 2009;15:2074-83
33. Bamiou DE, Free SL, Sisodiya SM, et al. Auditory interhemispheric transfer deficits, hearing difficulties, and brain magnetic resonance imaging abnormalities in children with congenital aniridia due to PAX6 mutations. Arch Pediatr Adolesc Med 2007;161:463-69

34. Webb EA, O'Reilly MA, Orgill J, et al. Rest-activity disturbances in children with septo-optic dysplasia characterized by actigraphy and 24-hour plasma melatonin profiles. J Clin Endocrinol Metab 2010;95:198-203

35. Ashkenazi-Hoffnung L, Lebenthal Y, Wyatt AW, et al. A novel lossof-function mutation in OTX2 in a patient with anophthalmia and isolated growth hormone deficiency. Hum Genet 2010;127:721-29

36. Tajima T, Ohtake A, Hoshino M, et al. OTX2 loss of function mutation causes anophthalmia and combined pituitary hormone deficiency with a small anterior and ectopic posterior pituitary. J Clin Endocrinol Metab 2009;94:314-19

37. Wyatt A, Bakrania P, Bunyan DJ, et al. Novel heterozygous OTX2 mutations and whole gene deletions in anophthalmia, microphthalmia and coloboma. Hum Mutat 2008;29:278-83

38. Schilter KF, Schneider A, Bardakjian T, et al. OTX2 microphthalmia syndrome: four novel mutations and delineation of a phenotype. Clin Genet 2011;79:158-68

39. Elliott J, Maltby EL, Reynolds B. Acase of deletion 14(q22.1->q22.3) associated with anophthalmia and pituitary abnormalities. J Med Genet 1993;30:251-52

40. Lemyre E, Lemieux N, Decarie JC, et al. Del(14)(q22.1q23.2) in a patient with anophthalmia and pituitary hypoplasia. Am J Med Genet 1998;77:162-65

41. Nolen LD, Amor D, Haywood A, et al. Deletion at 14q22-23 indicates a contiguous gene syndrome comprising anophthalmia, pituitary hypoplasia, and ear anomalies. Am J Med Genet A 2006; 140:1711-18

42. Hayashi S, Okamoto N, Makita Y, et al. Heterozygous deletion at 14q22.1-q22.3 including the BMP4 gene in a patient with psychomotor retardation, congenital corneal opacity and feet polysyndactyly. Am J Med Genet A 2008;146:2905-10

43. Bakrania P, Efthymiou M, Klein JC, et al. Mutations in BMP4 cause eye, brain, and digit developmental anomalies: overlap between the BMP4 and hedgehog signaling pathways. Am J Hum Genet 2008;82: $304-19$ 